\title{
Thermal non-equilibrium in coronal loops: A road to complex evolution
}

\author{
Daniel Müller ${ }^{1,2}$, A. De Groof ${ }^{3}$, V.H. Hansteen ${ }^{1}$ and H. Peter ${ }^{2}$ \\ ${ }^{1}$ Institute of Theoretical Astrophysics, University of Oslo, PO Box 1029 Blindern, 0315 Oslo, \\ Norway email: Daniel.Mueller@astro.uio.no \\ ${ }^{2}$ Kiepenheuer-Institut für Sonnenphysik, Schöneckstr. 6, 79104 Freiburg, Germany \\ ${ }^{3}$ Centre for Plasma Astrophysics, K.U. Leuven, Celestijnenlaan 200 B, 3001 Leuven, Belgium
}

\begin{abstract}
At high spatial and temporal resolution, coronal loops are observed to have a highly dynamic nature. Recent observations with SOHO and TRACE frequently show localized brightening "raining" down towards the solar surface. What is the origin of these features? Here we present for the first time a comparison of observed intensity enhancements from an EIT shutterless campaign with non-equilibrium ionization simulations of coronal loops in order to reveal the physical processes governing fast flows and localized brightening. We show that catastrophic cooling around the loop apex as a consequence of footpoint-concentrated heating offers a simple explanation for these observations. An advantage of this model is that no external driving mechanism is necessary as the dynamics result entirely from the non-linear character of the system.
\end{abstract}

The upper solar atmosphere, the transition region and corona, is highly complex and magnetically structured. Recent space observations with SOHO and TRACE have revealed that coronal loops, magnetically closed structures in the upper solar atmosphere, are intrinsically dynamic. Intensity enhancements ("blobs") are often seen to propagate along these loops, and these propagating disturbances are of great importance for coronal seismology and for the understanding of coronal heating mechanisms. Spectroscopic investigations show that these intensity enhancements have different signatures in UV spectral lines formed at different temperatures and exhibit Doppler shifts of the order of $50-100 \mathrm{~km} \mathrm{~s}^{-1}$.

Recently, De Groof et al. (2004a,b) observed propagating intensity enhancements in the He II $30.4 \mathrm{~nm}$ band with the Extreme-Ultraviolet Imaging Telescope (EIT), moving with up to $120 \mathrm{~km} \mathrm{~s}^{-1}$. After a detailed analysis, the authors ruled out slow magneto-acoustic waves as the origin of these intensity enhancements and instead identified them as flowing plasma blobs. As a result of these findings they favored the scenario of the "heatingcondensation cycle" as described in Müller et al. (2003, 2004) as a possible explanation. In that work, it was shown that localized brightening can be the result of catastrophic cooling of a loop which is predominantly heated at the footpoints. The heating leads to an evaporation of plasma into the coronal loop which then cools rapidly due to a loss of thermal equilibrium. The confined region of "condensed" plasma subsequently falls down under the effect of gravity in the form of a cool plasma blob.

In Müller et al. (2004) we described the evolution, speed and spectral signature of these traveling condensation regions, and pointed out a possible connection to the recent EIT observations. Compared to the loops described in this work, the loop structure seen in the data from the EIT shutterless campaign is larger and the observed blob velocities are higher, but the general features of the model encouraged us to carry out new simulations. For the models presented here, we adopted the loop geometry 
inferred from the EIT data and also used a higher heating rate to reach apex temperatures of around $3 \mathrm{MK}$ as suggested, e.g., by TRACE observations of large active region loops. For the first time we study the "heating-condensation cycle" in models of long active-region loops and find that the shocks caused by rapid cooling processes in hot loops can trigger further and more complex cooling events. The associated condensation regions, blobs of cool plasma with enhanced density and radiative losses, can be accelerated to much higher velocities than the usual condensation regions. While the first condensation region which is traveling down along the loop is strongly decelerated by the underlying loop plasma, a second condensation region is formed behind the first one. This trailing blob is traveling in the wake of the leading blob where the pressure is significantly lower. This reduced pressure results in
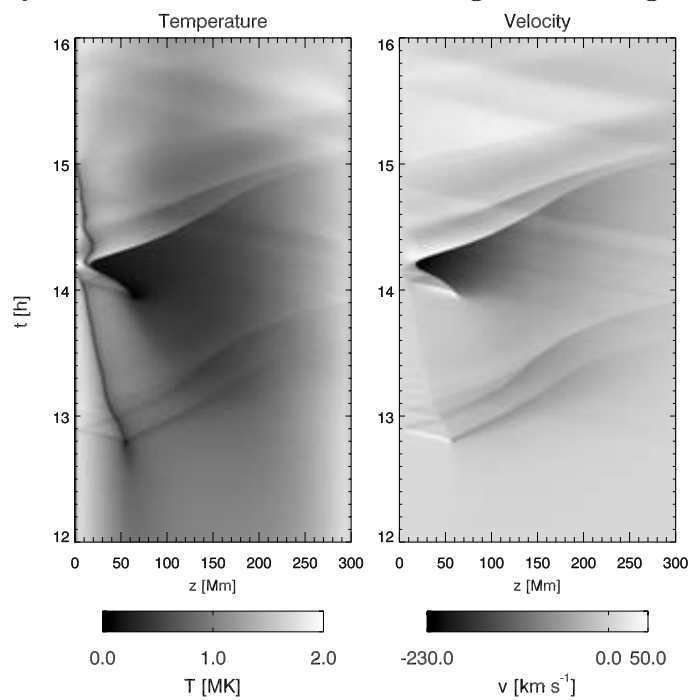

Figure 1: Temperature and velocity along the loop as a function of time. a plasma blob that is initially traveling with almost free-fall speed and can reach velocities of the order of $100 \mathrm{~km} \mathrm{~s}^{-1}$ before its deceleration sets in in the lower regions of the atmosphere.

In Fig. 1, temperature and velocity along the loop are displayed for a part of the simulation. The dark lanes in the temperature plot correspond to the cool plasma blobs, and the different slopes indicate different blob velocities. The velocity plot shows flow speeds of up to $230 \mathrm{~km} \mathrm{~s}^{-1}$, with the highest speeds occurring in the wake of the plasma blobs where the density is low. Fig. 2 displays the timedependent emission in the two spectral lines of $C I V(154.8 \mathrm{~nm})$ and $O V$ $(63.0 \mathrm{~nm})$ during the fall of the second condensation region. The profiles are integrated over the left half of the loop
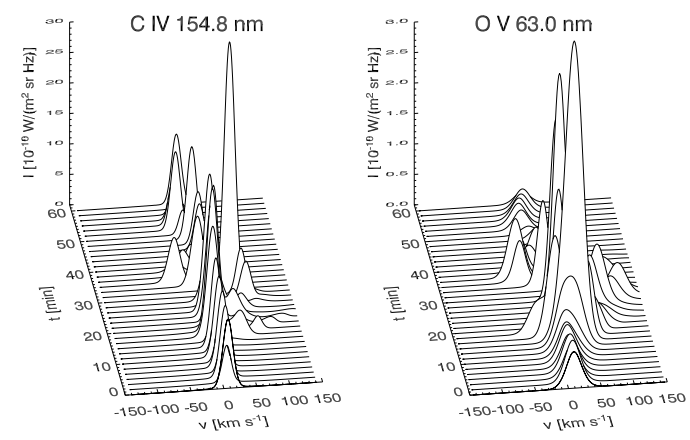
as seen from above. The plot shows Figure 2: Spectral signature of falling plasma the strong redshifts associated with the blobs in $C I V(154.8 \mathrm{~nm})$ and $O V(63.0 \mathrm{~nm})$. falling plasma and the transient brightening of the blob when it hits the transition region. For more details concerning the observations, we refer to the article of De Groof et al. (2004b, these proceedings).

\section{References}

De Groof, A., Berghmans, D., van Driel-Gesztelyi, L., \& Poedts, S. 2004a, A\&AA 415, 1141

De Groof, A., Müller, D. A. N., Berghmans, D. \& Poedts, S. 2004b, these proceedings

Müller, D. A. N., Hansteen, V. H., \& Peter, H. 2003, A\&A 411, 605

Müller, D. A. N., Peter, H., \& Hansteen, V. H. 2004, $A \mathscr{E} A$, 424, issue I 